\title{
GABA the Hutt
}

\author{
Friedrich C. Luft
}

Received: 2 April 2014 / Accepted: 2 April 2014 /Published online: 16 April 2014

(C) Springer-Verlag Berlin Heidelberg 2014

The Hutts were a sentient species of large gastropods with short arms, wide cavernous mouths, and huge eyes, who controlled a large space empire in Hutt Space. We encountered a famous Hutt (JABBA) in Star Wars I. Whether or not Hutts existed or functioned as claimed is unclear. Is GABA turning into a Hutt? $\gamma$-Aminobutyric acid (GABA) is the main inhibitory neurotransmitter in the mammalian central nervous system. GABA participates in regulating neuronal excitability. In humans, GABA is also directly responsible for the regulation of muscle tone. Although in chemical terms GABA is an amino acid, GABA is rarely called an amino acid because the term "amino acid," used without a qualifier, by convention refers to the alpha amino acids, which GABA is not. GABA is never incorporated into proteins (Fig. 1).

In spastic diplegia syndromes, nerves damaged from the condition's upper motor neuron lesion exhibit impaired GABA absorption, which leads to hypertonia of muscles signaled by those nerves that can no longer absorb GABA. GABA acts at inhibitory synapses in the brain by binding to specific transmembrane receptors in the plasma membrane of both pre and postsynaptic neurons. The binding causes ion channels to open, allowing the flow of either negatively charged chloride ions into the cell, or positively charged potassium ions out of the cell. This action results in a negative change in the transmembrane potential, usually causing hyperpolarization. Two general classes of GABA receptor are known: the GABA-A receptor is part of a ligand-gated ion channel complex; the GABA-B receptor is a $G$ proteincoupled receptor that opens or closes ion channels via Gprotein intermediaries. Neurons producing GABA as their

F. C. Luft $(\bowtie)$

Experimental and Clinical Research Center, Charité Medical Faculty and the Max-Delbrück Center for Molecular Medicine, Lindenbergerweg 80, 13125 Berlin, Germany

e-mail: luft@mdc-berlin.de output are called GABAergic neurons. GABA receptors are important drug targets, for instance for benzodiazepines and barbiturates. Baclofen is a derivative of GABA that is primarily used to treat spasticity and is in the early research stages for use in the treatment of alcohol withdrawal.

Thus far, we have solely discussed neurons. However, is GABA of any importance elsewhere? To start at the beginning, perhaps we should consult embryonic stem (ES) cells. Teng et al. detected the repertoire of components for GABA synthesis, storage, reaction, and termination in ES cells and embryonal carcinoma stem cells by biological assays and then directly quantified released GABA in the intercellular milieu from ES cells by chemical assays [1]. They found that ES cells processed GABAergic circuit machinery and spontaneously released GABA, which suggests that ES cells could autonomously establish a GABA niche via release of the transmitter. Could GABA be important in fat (outside the brain of course)? Choi et al. studied Wnt5A-mediating neurogenesis of human adipose tissue-derived stem cells in a 3D microfluidic cell culture system. Their data showed that 3D microfluidic cell culture induces activation of the Wnt $5 \mathrm{~A} / \beta$-catenin signaling pathway [2].

In neural and nonneural tissues, three enzymes metabolize substrates to produce GABA: glutamic acid decarboxylase (GAD), which produces GABA from glutamic acid, GABAtransaminase (GABA-T), and succinic semialdehyde dehydrogenase (SSADH) [3]. Production of succinic acid by SSADH allows entry of the GABA carbon skeleton into the tricarboxylic acid cycle. Alternative sources of GABA include putrescine, spermine, spermidine, and ornithine, which produce GABA from deamination and decarboxylation reactions. GAD from mammalian brain occurs in two molecular forms. The two forms are the product of different genes, differing in nucleotide sequence, immunoreactivity, and subcellular localization. GAD and GABA have been investigated in many nonneural tissues including liver, kidney, pancreas, testis, 
a<smiles>NCC(=O)O</smiles>

b<smiles>N[14CH2][14CH2][14CH2]C(=O)O</smiles>

Glycine

GABA

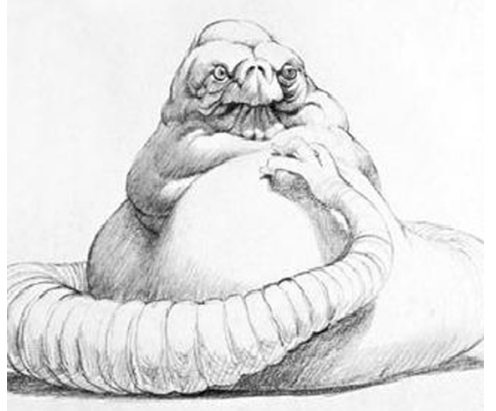

$\alpha$-amino acids (generic formula $\mathrm{H} 2 \mathrm{NCHRCOOH}$ ) in most cases. GABA (b), although in chemical terms is an amino acid, does not meet these criteria, nor is it incorporated into proteins. We include concept artwork of Jabba the Hutt designed for "Return of the Jedi" by Industrial Light \& Magic http://en. wikipedia.org/wiki/Jabba_the_Hutt

exhibit sodium-dependent GABA uptake. Platelets have also been used as a model for GABAergic mechanisms in the brain. They studied 13 patients with childhood absence epilepsy and sex- and age-matched controls. The patients had lower GABA-T activity than controls. The capacity of GABA uptake into the platelets was higher in patients using the antiepileptic drug, valproate, but not in those treated with a second compound, ethosuximide. The affinity of the

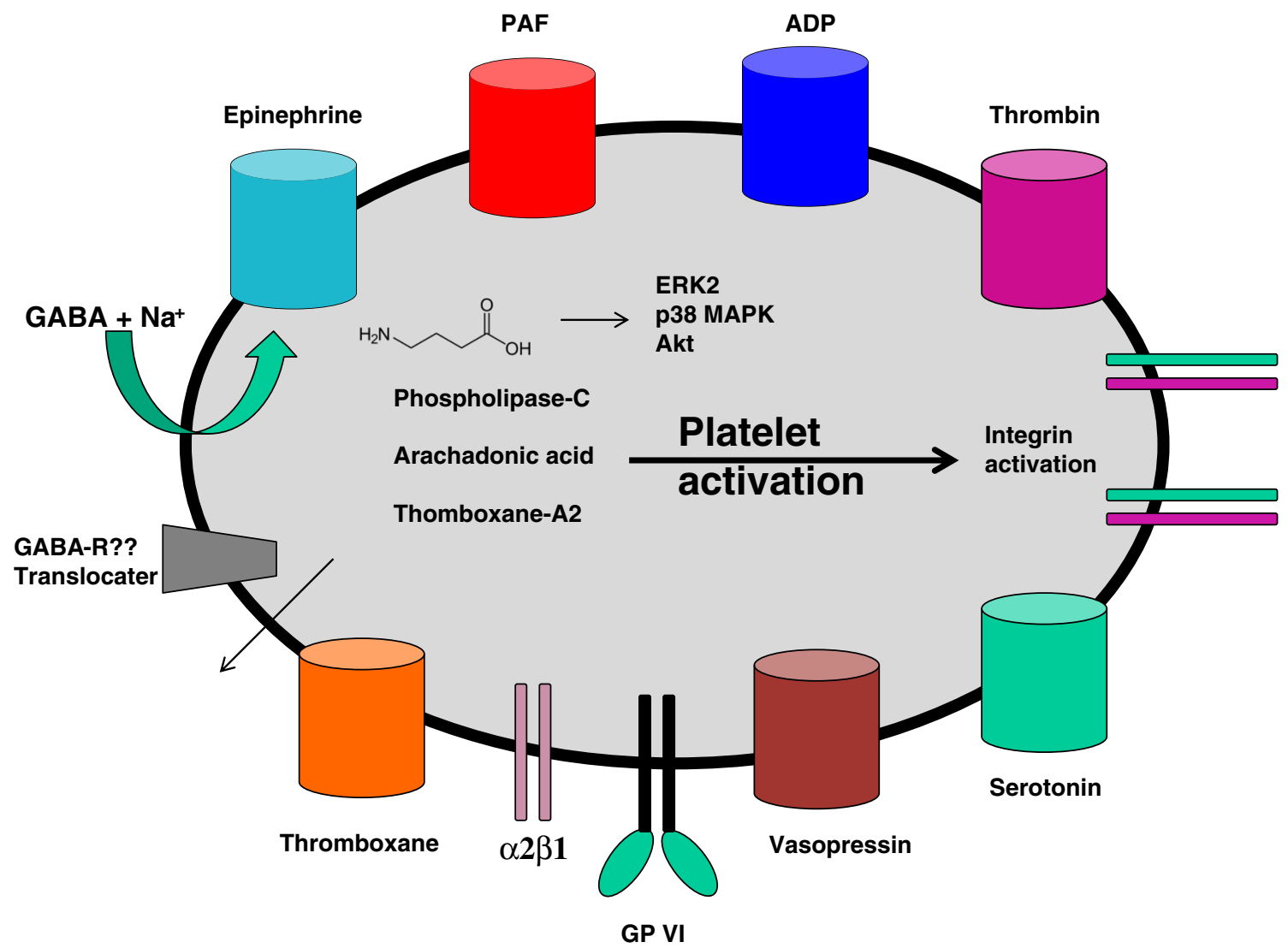

Fig. 2 The platelet is a "grand central station" of signaling and only a small part is shown here. The authors propose that GABA is introduced through a sodium-dependent mechanism. GABA receptors have not been found on platelets, although a translocator protein has been proposed. $P A F$

platelet-activating factor, $A D P$ adenosine diphosphate, GP VI glycoprotein VI that interacts with collagen, $E R K$ extracellular-related kinase, $M A P K$ mitogen-activated protein kinase 
transporters was not altered. The authors believe that the observed peripheral alterations may indicate impaired function of brain GABAergic systems in children with absence epilepsy.

Lin et al. in this issue of J Mol Med also focused on platelets [6]. They reasoned that since platelets take up GABA and as they harbor binding sites for benzodiazepines that seem to inhibit platelet aggregation, platelet function could be influenced by GABAergic mechanisms. Lin et al demonstrate that GABA is abundant in platelets. The authors found that GABA specifically inhibited collagen-induced platelet activation accompanied by $\left[\mathrm{Ca}^{2+}\right]_{\mathrm{i}}$ mobilization, phospholipase $\mathrm{C} \gamma 2$, protein kinase $\mathrm{C}$, Akt phosphorylation, and hydroxyl radical formation. They also observed that GABA interfered with fluorescein isothiocyanate-collagen binding to platelet membranes and produced a concentration-dependent shift in the collagen platelet-activating concentration-response curve. The finding suggests competitive platelet inhibition. GABA inhibited platelet activation by convulxin, a glycoprotein VI agonist. Instead, activation induced by the integrin $\alpha 2 \beta 1$ agonist, aggretin, was not inhibited. Immunoprecipitation and surface plasmon resonance revealed that GABA bound directly to glycoprotein VI on platelets. In functional experiments, the closure time of whole blood and the occlusion time of platelet plug formation were significantly prolonged by GABA in vivo. The implication of their findings is that GABA could be a specific inhibitor of collagen glycoprotein VI and may be involved in an endogenous negative feedback mechanism for platelet activation. The authors suggest that GABA may represent a potential target for the development of novel interventions for the treatment of cardiovascular diseases associated with platelet activation, such as stroke and myocardial infarction. The platelet (Fig. 2) is a busy place with numerous interacting components, many of which are already being addressed by pharmacological agents.

The authors suggest that GABA specifically inhibited collagen-induced platelet aggregation. Collagen is present in the vascular subendothelium and vessel wall and acts as both a substrate for platelet adhesion and an endogenous platelet activator. Integrins such as $\alpha 2 \beta 1$ and glycoprotein VI are platelet receptors that are known to interact directly with collagen. How could the authors' findings be translated into a clinically relevant result? GABA receptors have never been reported on platelets. To what degree the authors looked for GABA receptors is not outlined in this report. The inhibitory effect of GABA-operative pharmaceuticals is said to occur via an $18-\mathrm{kDa}$ translocator protein, which has been termed a "peripheral-type" benzodiazepine receptor. Rajtar et al. studied the effects of diazepam and clonazepam on platelets [7]. They reported that in vitro diazepam, and in a much smaller degree clonazepam, may downregulate platelet activation and release of some proinflammatory mediators and speculated that the effects were probably exerted by specific benzodiazepine-binding sites. However, no such binding sites were characterized. Bazziczi et al. studied peripheral benzodiazepine receptors on platelets of fibromyalgic patients [8]. However, their characterizations were solely based on radioligand-binding studies and no actual receptor was delineated. Thus, any translation of the interesting findings reported by Lin et al. [6] will have to await more extensive pharmacological investigations.

Respectfully, Friedrich C. Luft

\section{References}

1. Teng L, Tang YB, Sun F, An SM, Zhang C, Yang XJ, Lv HY, Lu Q, Cui YY, Hu JJ et al (2013) Non-neuronal release of gammaaminobutyric acid by embryonic pluripotent stem cells. Stem Cells Dev 22:2944-2953

2. Choi J, Kim S, Jung J, Lim Y, Kang K, Park S, Kang S (2011) Wnt5amediating neurogenesis of human adipose tissue-derived stem cells in a 3d microfluidic cell culture system. Biomaterials 32:7013-7022

3. Tillakaratne NJ, Medina-Kauwe L, Gibson KM (1995) Gammaaminobutyric acid (gaba) metabolism in mammalian neural and nonneural tissues. Comp Biochem Physiol A Physiol 112:247-263

4. Sherif FM (1994) Gaba-transaminase in brain and blood platelets: basic and clinical aspects. Prog Neuro-Psychopharmacol Biol Psychiatry 18:1219-1233

5. Rainesalo S, Eriksson K, Saransaari P, Keranen T (2004) Uptake of gaba and activity of gaba transaminase in blood platelets from children with absence epilepsy. Neurochem Res 29:1873-1877

6. Lin KH, Lu WJ, Wang SH, Fong TH, Chou DS, Chang CC, Chang NC, Chiang YC, Huang SY, Sheu JR (2014) Characteristics of endogenous gamma-aminobutyric acid (gaba) in human platelets: functional studies of a novel collagen glycoprotein VI inhibitor. J Mol Med. doi: 10.1007/s00109-014-1140-7

7. Rajtar G, Zolkowska D, Kleinrok Z (2002) Effect of diazepam and clonazepam on the function of isolated rat platelet and neutrophil. Med Sci Monit Int Med J Exp Clin Res 8:PI37-PI44

8. Bazzichi L, Giannaccini G, Betti L, Italiani P, Fabbrini L, Defeo F, Giacomelli C, Giuliano T, Rossi A, Uccelli A et al (2006) Peripheral benzodiazepine receptors on platelets of fibromyalgic patients. Clin Biochem 39:867-872 